\title{
A simple strategy based on fibers coated with surfactant-functionalized multiwalled carbon nanotubes to improve the properties of solid-phase microextraction of phenols in aqueous solution
}

\author{
Xueqing Zhou ${ }^{1,2^{*}} \mathbb{D}$, Yanli Xie ${ }^{1}$, Zhendong Zhao ${ }^{1}$ and Wenyan Fu²
}

\begin{abstract}
Methods and experiments: In this study, a functionalized multiwalled carbon nanotube (MWCNT)-coated solidphase microextraction (SPME) fiber was developed for concentrating analytes in aqueous samples. Sodium deoxycholate (NaDC) was used as a dispersing agent for non-covalent modification of MWCNTs. The coating showed porous structure and large adsorption capacity. To investigate the capability of this MWCNTs/NaDC SPME fiber, it was applied to the analysis of phenols in aqueous solution. After extraction, the analytes were desorbed in an acetonitrile-water solution and analyzed using high-performance liquid chromatography.

Results: The MWCNTs/NaDC fiber exhibited good analytical performance, and fine preparation reproducibility was obtained with the relative standard deviations (RSDs) ranging from $4.9 \%$ to $10.2 \%(n=6)$ in one batch, from $5.7 \%$ to $11.9 \%(n=3)$ among different batches. Under the optimum extraction conditions, the detection limits were $0.15-0.30 \mathrm{ng} / \mathrm{mL}(\mathrm{S} / \mathrm{N}=3)$, the linear detection ranges were $1-100 \mathrm{ng} / \mathrm{mL}\left(R^{2} \geq 0.9997\right)$ for these analytes, and good recoveries (80.3-95.4\%) were obtained for the spiked samples.
\end{abstract}

Conclusion: This is a simple and accurate pretreatment method for the analysis of phenols in aqueous samples.

Keywords: Functionalized multiwalled carbon nanotube, Solid-phase microextraction, Surfactant, Phenol

\section{Introduction}

Phenols are hydroxyl-containing derivatives of aromatic hydrocarbons, which are one of very toxic organic contaminants $[1,2]$. Due to the dense population and industry, the phenols pollution in environment is becoming increasingly serious [3]. Monitoring of phenols contaminants is particularly important, which is the basis and prerequisite for the control and remediation of phenols. Some conventional methods, such as Liquid-liquid

\footnotetext{
*Correspondence: zhouxq001@hainanu.edu.cn

${ }^{1}$ Analytical and Testing Center, Hainan University, Haikou 570228, China Full list of author information is available at the end of the article
}

extraction and solid-phase extraction, were often used to extract analytes in aqueous samples. However, these techniques were usually time-consuming and required toxic organic solvents $[4,5]$. Therefore, ideal samplepreparation techniques are commonly required.

In the past few years, solid-phase microextraction (SPME) have been developed for the extraction of phenols [6-9] The obvious advantages of SPME are solvent-free process, simplicity of operation, and a short extraction time, which reduces contamination of the sample and loss of analytes [10, 11]. Besides, it can combine sampling, extraction and enrichment into a single step [12]. In these methods, commercial non-polar 
polydimethylsiloxane (PDMS) coated SPME fibers are widely used for extraction of phenols in environmental water samples [13, 14]. For instance, Quintana et al. [15] and Montero et al. [16] applied PDMS coated SPME to extract phenols from environmental samples. Yu et al. [17] selected commercial PDMS/DVB fiber for the determination of phenols and related chlorophenols in water. However, due to the polarity of phenols, the application of PDMS fibers has to be accompanied with derivatization, which increases the triviality of pretreatment. And their performance is not always satisfactory for the extraction of large numbers of varied analytes due to their thermal instability or limited selectivity [18]. To obtain high extraction efficiencies for these compounds, various types of SPME coatings have been investigated, including molecularly imprinted polymers [19], ionic liquids [20], metal-organic frameworks [21], and carbon materials [22-25].

Multiwalled carbon nanotubes (MWCNTs) have unique electronic, mechanical, and chemical properties and have attracted attention in recent years [26-30] MWCNTs contain internal cavities that are large enough to allow analytes to penetrate, and their surfaces and interspaces within nanotube bundles provide excellent sorption [31]. Because of their unique properties and their hydrophobic character, MWCNTs are superior adsorbents for aliphatic hydrocarbons [32], polycyclic aromatic hydrocarbons (PAHs) [33], phthalates [34], and volatile organic compounds [35, 36]. Jiang et al. [37] first employed CNTs as SPE adsorbent for the determination of bisphenol A, 4-nonylphenol and 4-tert-octylphenol, which showed good performance. In addition, functionalization of the CNTs plays a key role in selectivity for polar compounds. Hu et al. [38] synthesized amino modified multi-walled carbon nanotubes/polydimethylsiloxane (MWCNTs-DDM/PDMS) coating for stir bar sorptive extraction, which was successfully applied to the analysis of phenols in environmental water and soil samples. Ai et al. [39] reported an ionic liquid functionalized multiwalled carbon nanotubes-polyaniline (MWCNT@ IL/PANI) nanocomposite coating. This coating exhibited high extraction efficiency.

Moreover, it was reported that surfactants or macromolecules could interact strongly with MWCNTs, which have an effect on their structures and polarity, and modification of MWCNTs with surfactants or macromolecules can increase their solubility and dispersibility $[40,41]$. It can be speculated that surfactants modified MWCNTs will be an efficient SPME coating material for the extraction of phenols because of the possible interaction between surfactants modified MWCNTs and the target phenols including hydrophobic interaction and intermolecular hydrogen bond.
In this study, a new sodium deoxycholate functionalized multiwalled carbon nanotube-coated (MWCNTs/ $\mathrm{NaDC}$ ) fiber was fabricated, and was used to analysis of phenols from environmental samples. The combination of SPME with high-performance liquid chromatography (HPLC) provides an accurate and sensitive method for the determination of phenols in aqueous solution, and was applied to seawater samples from the South China Sea and Wastewater.

\section{Experimental}

\section{Reagents and materials}

Phenol, $p$-nitrophenol (4-NP), $o$-nitrophenol (2-NP), 2, 4-dimethylphenol (2,4-DMP), and 2,4-dichlorophenol (2,4-DCP) standards were purchased from Sinopharm Chemical Reagent Co., Ltd. (Shanghai, China). Hydrogen nitrate (AR grade, purity 65-68\%) and sulfuric acid were purchased from Guangzhou Chemical Reagents (Guangzhou, China). HPLC-grade acetonitrile (ACN), methanol, isopropanol, and formic acid were from Thermo Fisher Scientific Co. (Waltham, MA). Fused-silica fibers (120 $\mu \mathrm{m}$ i.d.) were obtained from Ruifeng Chromatographic Device Co. Ltd (Yongnian, China). MWCNTs (20-30 nm, purity $>98 \%$ mass fraction) were purchased from Sigma-Aldrich (St. Louis, MO). SPME hand shank and PDMS/DVB fiber $(1 \mathrm{~cm}$ length, $65 \mu \mathrm{m}$ thick, Supelco, USA) were purchased from Sigma-Aldrich Co., Ltd. (Shanghai, China). Acrylic ester was obtained from Guangzhou Chemical Reagents. Sodium deoxycholate (NaDC) was purchased from Aladdin Chemistry (Shanghai, China). Ultrapure water was prepared with a Milli-Q water purification system (Millipore, Bedford, MA). All other chemicals were of analytical grade.

\section{Instruments and conditions}

Sample analyses were carried out using a Waters (Milford, MA) e2695 system equipped with a 2998 photodiode array detector. A C18 column $(250 \mathrm{~mm} \times 4.6 \mathrm{~mm}$ i.d., $5 \mu \mathrm{m}$ particle size, Agela) was used for the chromatographic separation. The mobile phase was a mixture of $0.2 \%$ acetic acid (A) and acetonitrile (B) at a flow rate of $1.0 \mathrm{~mL} / \mathrm{min}$. We used the following gradient elution: 0-5 min, $A=70 \%$ and $B=30 \% ; 20 \mathrm{~min}, A=20 \%$ and $B=80 \% ; 21 \mathrm{~min}, \mathrm{~A}=10 \%$ and $\mathrm{B}=90 \% ; 25 \mathrm{~min}, \mathrm{~A}=10 \%$ and $\mathrm{B}=90 \%$; then the ratio of solvent $\mathrm{B}$ decreased to $30 \%$ in $2 \mathrm{~min}$ and kept for $3 \mathrm{~min}$ to equilibrate the column. The total run time was about $25 \mathrm{~min}$. The column temperature was $30^{\circ} \mathrm{C}$ and the wavelength was $280 \mathrm{~nm}$.

Transmission electron microscope (TEM) images were recorded using a JEM 2100 instrument (JOEL, Tokyo, Japan). SEM observation was executed using a Hitachi S-3000 N(Japan) scanning electron microscope after fixing the samples on a brass holder and coating them with 
gold. Fourier transform infrared (FT-IR) spectra of the materials were obtained on a TENSOR 27 spectrometer (Bruker, Ettlingen, Germany). The freeze-dried samples were mixed with $\mathrm{KBr}$ compressed into semitransparent $\mathrm{KBr}$ pellets before the measurement. XRD spectra were recorded on a D8 Advance X-ray diffractometer ((Bruker, Germany) with $\mathrm{Cu}-\mathrm{K}$ a radiation $(\lambda=0.154 \mathrm{~nm})$. The XRD was operated at $40 \mathrm{kV}$ and $40 \mathrm{~mA}$ in a step scan mode. The scanning speed was $0.025^{\circ} \mathrm{s}$. XRD measurements were performed over a $2 \theta$ range of $10^{\circ}-40^{\circ}$.

\section{Synthesis of functionalized MWCNTs}

Functionalized MWCNTs (MWCNTs/NaDC) were synthesized as follows. First, $2 \mathrm{~g}$ of pristine MWCNT powder was added to $100 \mathrm{~mL}$ of a mixture of $\mathrm{HNO}_{3}$ and $\mathrm{H}_{2} \mathrm{SO}_{4}$ $(1: 3, \mathrm{v} / \mathrm{v})$. The mixture was then heated in a water bath at $80{ }^{\circ} \mathrm{C}$ for $3 \mathrm{~h}$ with stirring. The processed MWCNTs were collected by filtration and washed with deionized water until the $\mathrm{pH}$ approached neutral, and then dried at $60{ }^{\circ} \mathrm{C}$ for further use. Second, the processed MWCNTs were modified by sodium deoxycholic acid $(\mathrm{NaDC})$ with a mass ratio of 1:1 (see Additional file 1). The suspension was ultrasonic for $30 \mathrm{~min}$ in the ultrasonic crushing instrument, and then by magnetic stirring for $12 \mathrm{~h}$. The resulting solution was filtered, washed with ultrapure water, and dried at $60{ }^{\circ} \mathrm{C}$ for $24 \mathrm{~h}$. Figure 1 presents the synthetic strategy for the MWCNTs/NaDC.

\section{Preparation of the MWCNTs/NaDC SPME fiber}

The MWCNTs/NaDC SPME fiber was fabricated using the following processes. First, a silica fiber was cut to $10 \mathrm{~cm}$. Then, one end $(2.0 \mathrm{~cm})$ was burned on an alcohol blast burner to remove the protecting polyimide layer. Subsequently, the fiber was washed thoroughly with $10 \mathrm{~mL}$ of water and $10 \mathrm{~mL}$ of acetonitrile in sequence, and dried at $60{ }^{\circ} \mathrm{C}$ for $12 \mathrm{~h}$. Next, the fiber was parallelly dipped in epoxy glue for $30 \mathrm{~s}$, and then coated. The unnecessary epoxy glue was removed with a tweezers to generate a homogeneous film of glue. And the equal in quality of MWCNT/NaDC was immobilized on every silica fiber by silicone glue. The coated section was $2.0 \mathrm{~cm}$ long. Finally, the proposed SPME fiber was heated to $200{ }^{\circ} \mathrm{C}$ for $5 \mathrm{~h}$ to remove any contaminants.

For comparison, the preparation procedure for MWCNTs fiber was the same as that for the MWCNTs/NaDCcoated SPME fiber as described above.

\section{SPME procedure}

For phenol analysis, all extraction experiments were carried out in a $10-\mathrm{mL}$ working solution, which was introduced to a $20-\mathrm{mL}$ amber vial capped with a polytetrafluoroethylene-coated septum. The solution was agitated at $1100 \mathrm{rpm}$ by magnetic stirring with a Tefloncoated stir bar. To perform the extraction, the MWC$\mathrm{NTs} / \mathrm{NaDC}$-coated SPME fiber was immersed in a water sample for a certain time. After extraction, the fiber was removed and placed in a $5-\mathrm{mL}$ vial. Then, the analytes were desorbed in a $2 \mathrm{~mL}$ solution of $70 \% \mathrm{ACN}$ solvent, and $10 \mu \mathrm{L}$ of the stripping solvent was used for HPLC analysis. The chromatographic peak area was used to evaluate the extraction efficiency under different conditions. The MWCNTs/NaDC SPME fiber could be reused after rinsing with the $70 \% \mathrm{ACN}$ solvent. To avoid memory effects, the SPME fibers were desorbed in a $70 \% \mathrm{ACN}$ solvent after extraction until the desorption solution had a flat baseline on injection into the HPLC.

\section{Analysis of real samples}

Water samples were collected from the South China Sea near Baishamen, Yangpu, and Holiday Beach. Wastewater sample were collected from a chemical plant. These samples were analyzed immediately after sampling without any pretreatment.

\section{Repeatability tests}

All experiments including the optimization of extraction conditions and the real samples analysis were performed in triplicate. The chromatographic peak area was used for quantification, and the experimental results were expressed as mean \pm standard deviation.

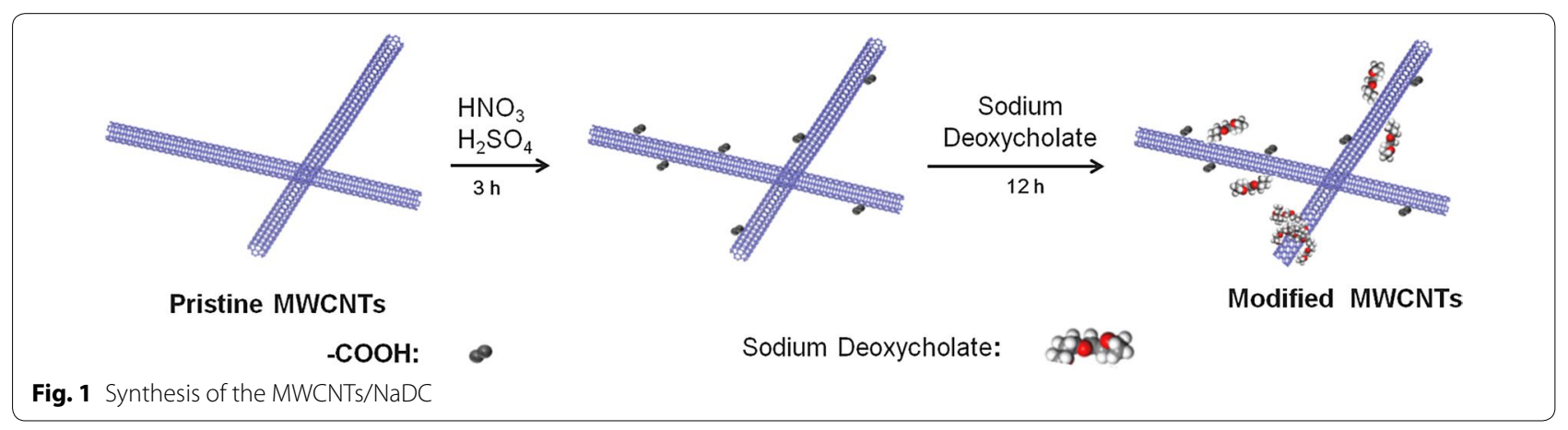



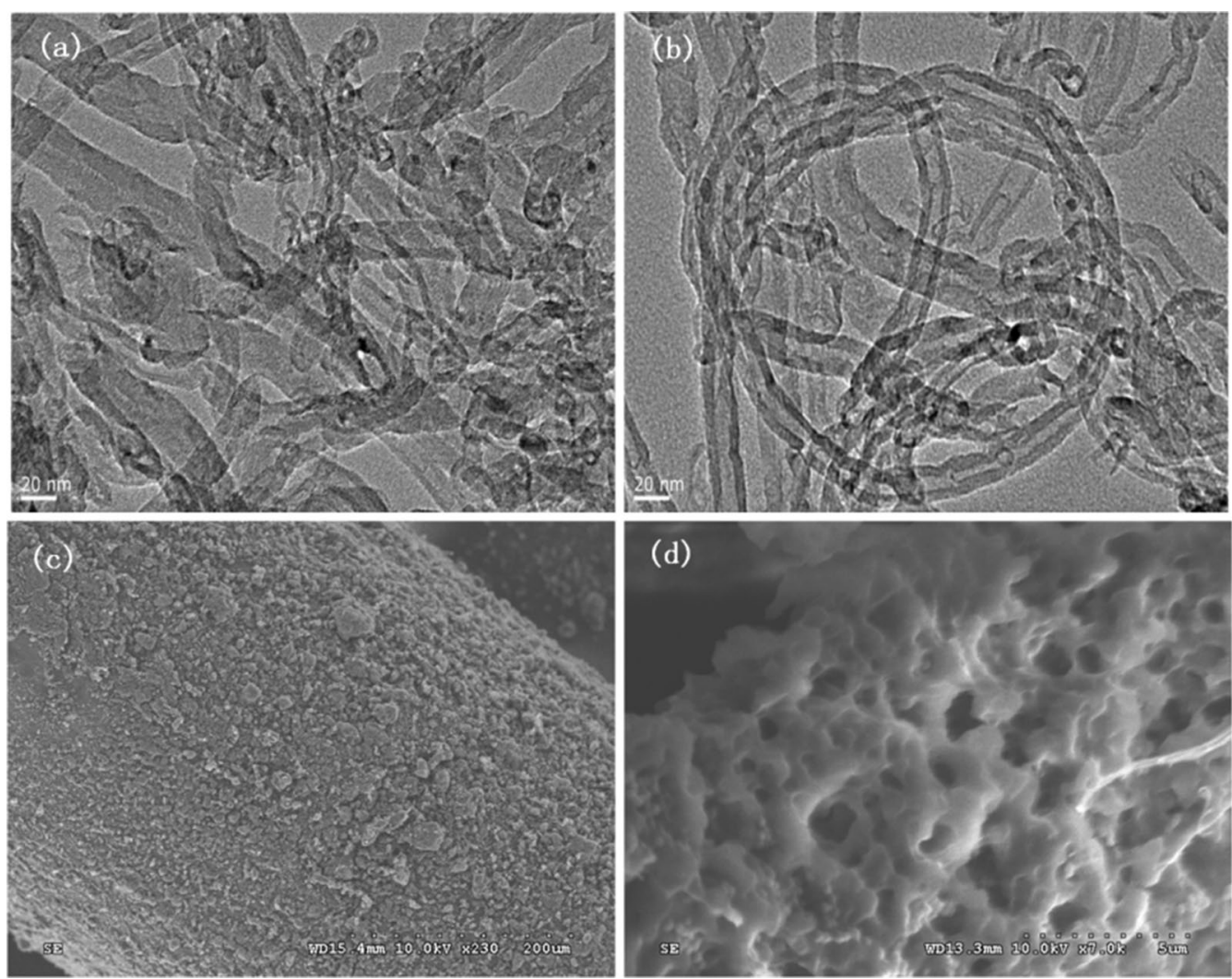

Fig. 2 TEM image of the original MWCNTS (a), TEM image of the modified MWCNTs after processing with NaDC (b), and SEM image of the MWCNTs/NaDC coating surface of the silica fiber $(\mathbf{c}, \mathbf{d})$

\section{Results and discussion}

\section{Preparation and characterization of modified MWCNTs}

The TEM and SEM images of the coating are shown in Fig. 2. As can be seen, the TEM image of the original MWCNTs (Fig. 2a) showed an aggregated structure, whereas the MWCNTs/NaDC (Fig. 2b) was well-dispersed in coating. SEM image reveal the presence of MWCNTs/NaDC coating the surface of the silica fiber in Fig. 2c. The coating presents net-like and porous structure, and the coating thickness was approximately $20 \mu \mathrm{m}$ (Fig. 2d). The MWCNTs/NaDC fiber had high specific surface area, which was favorable for the adsorption/ extraction of analytes. So it could be concluded that the introduction of $\mathrm{NaDC}$ could avoid the aggregation of MWCNTs and improve the dispersion of MWCNTs on SPME fiber.

The FT-IR spectra of MWCNTs/NaDC and original MWCNTs are shown in Fig. 3. The absorption peak at $\sim 1720 \mathrm{~cm}^{-1}$ was the characteristic peaks of stretching vibration of the $\mathrm{C}=\mathrm{O}$ in the spectral curve of MWCNTs/NaDC. The absorbance peaks at approximately $1169 \mathrm{~cm}^{-1}$ assigned to the $\mathrm{C}-\mathrm{OH}$ stretch. After $\mathrm{NaDC}$ modification, because of the interaction between

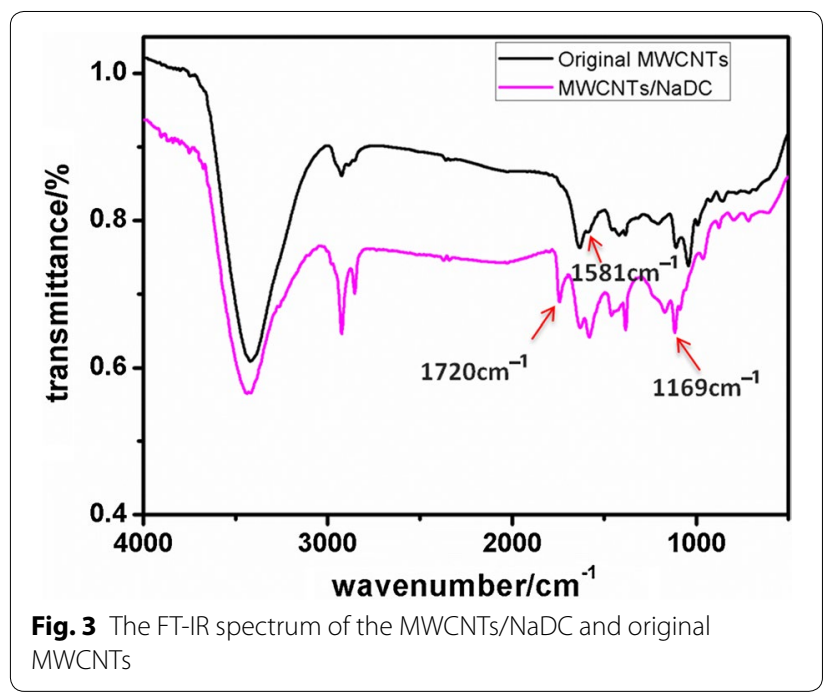

NaDC with MWCNTs, the stretching vibration of $\mathrm{C}=\mathrm{C}\left(\sim 1581 \mathrm{~cm}^{-1}\right)$ peak move higher wavenumber [42]. The result showed that $\mathrm{NaDC}$ is mainly combined with the surface of MWCNTs-COOH by random adsorption in a non-covalent modification way. 


\section{Enrichment factor of SPME}

For the equilibrium adsorption experiments, the enrichment factor of SPME depended on the interactions (i.e., $\pi-\pi$, hydrogen bonding, and electrostatic) between the analytes and MWCNTs/NaDC. The enrichment factor $(\mathrm{EF})$ is defined as the ratio of the extracted ananlyte with regard to the initial amount. And the chromatographic peak area was used for quantification of the final analyte concentration after extraction, which was obtained by direct injection of $10 \mu \mathrm{L}$ of standard solution.

The enrichment factor $(E F)$ was calculated as follows:

$$
E F=\frac{C_{A}}{C_{i}}
$$

where $C_{\mathrm{A}}$ was the amount of analyte extracted by SPME, $C_{\mathrm{i}}$ is the initial concentration.

The SPME using the MWCNTs/NaDC fiber was carried out with phenols concentration of $10.0 \mathrm{ng} / \mathrm{mL}$. The results are represented in Table 1 . As can be seen, the MWCNTs/NaDC fiber presents high EF values for five phenols. It has been reported that the van der Waals force, $\mathrm{H}$-bonding interactions from solutes as hydrogenbonding donors, and followed by $\pi$-electron polarizability, may play important roles on the adsorption of phenols by MWCNTs in the aqueous environment [43].

\section{Effect of experimental conditions on the extraction and desorption with the MWCNTs/NaDC SPME fiber}

To evaluate the performance of the MWCNTs/NaDC SPME fiber, different experimental parameters that could affect extraction efficiency, including the extraction time, the inorganic salt concentration, the composition of the elution solvent, and desorption time, were investigated and optimized.

\section{Extraction time}

Extraction time is an important factor that influences the extraction efficiency, and there is a correlation between the extraction amount and the extraction time. The effect of extraction time on the extraction efficiency of the MWC$\mathrm{NTs} / \mathrm{NaDC}$ coating was studied by varying the extraction time from $10 \mathrm{~min}$ to $60 \mathrm{~min}$. Extraction profiles for the five phenols are shown in Fig. 4a. The results indicated that the extraction efficiency increased with the exposure time, and the extraction equilibrium was reached in $20 \mathrm{~min}$ for 2,4DCP, $30 \mathrm{~min}$ for 2,4-DMP, $40 \mathrm{~min}$ for phenol and 4-NP, and $50 \mathrm{~min}$ for 2-NP. All phenols reached extraction equilibrium within $50 \mathrm{~min}$. Therefore, $50 \mathrm{~min}$ was chosen as the preferred extraction time.

\section{lonic strength}

Ionic strength is another parameter influencing the extraction efficiency of analytes in an aqueous solution.

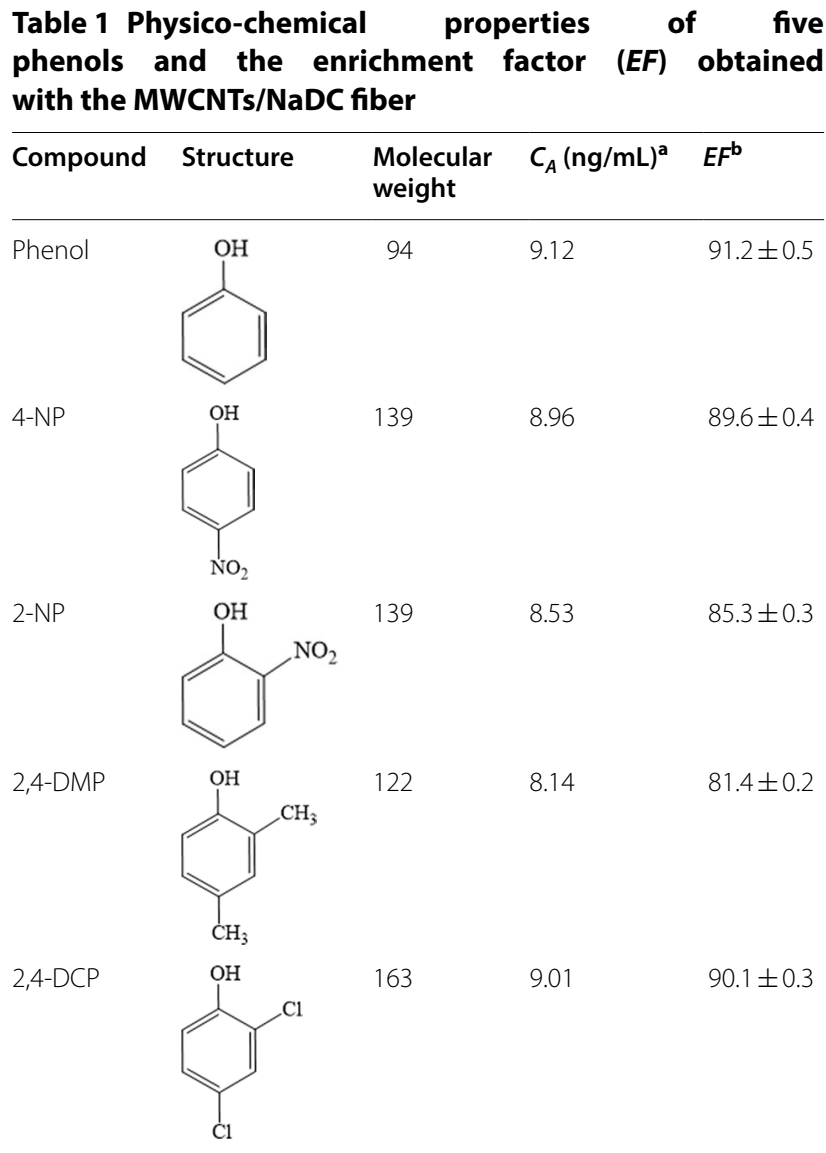

Samples of South China seawater $(10 \mathrm{~mL})$ were spiked with phenols at $10.0 \mathrm{ng} /$ $\mathrm{mL}$ and extracted

${ }^{a} C_{A}$ was calculated as the concentration of the analyte extracted

b The enrichment factor $(E F)$ of the analytes was calculated as the ratio of the analyte concentration after extraction to that in the original sample

Addition of inorganic salt can decrease the solubilities of analytes in an aqueous sample and enhance their concentrations in the adsorbent. Therefore, the influence of ionic strength was investigated by adding different proportions of $\mathrm{NaCl}(0-36 \% \mathrm{w} / \mathrm{v})$ as a salting-out agent (Fig. $4 \mathrm{~b})$. The results showed that most compounds had higher extraction efficiencies with high salt concentrations than with low salt concentrations, and $36 \%(\mathrm{w} / \mathrm{v}) \mathrm{NaCl}$ was chosen for subsequent extractions.

\section{Composition of the elution solvent}

The effect of the composition of the elution solvent on desorption was also investigated (Fig. 4c). A mixture of $\mathrm{ACN}$ and water gave good solubility for the phenols on the modified MWCNTs fiber. Generally, as the water volume fraction increased, the amount of desorption also increased for most phenols. The optimum desorption was achieved with an ACN volume fraction of $70 \%$. Consequently, 70\% ACN was used in subsequent experiments. 

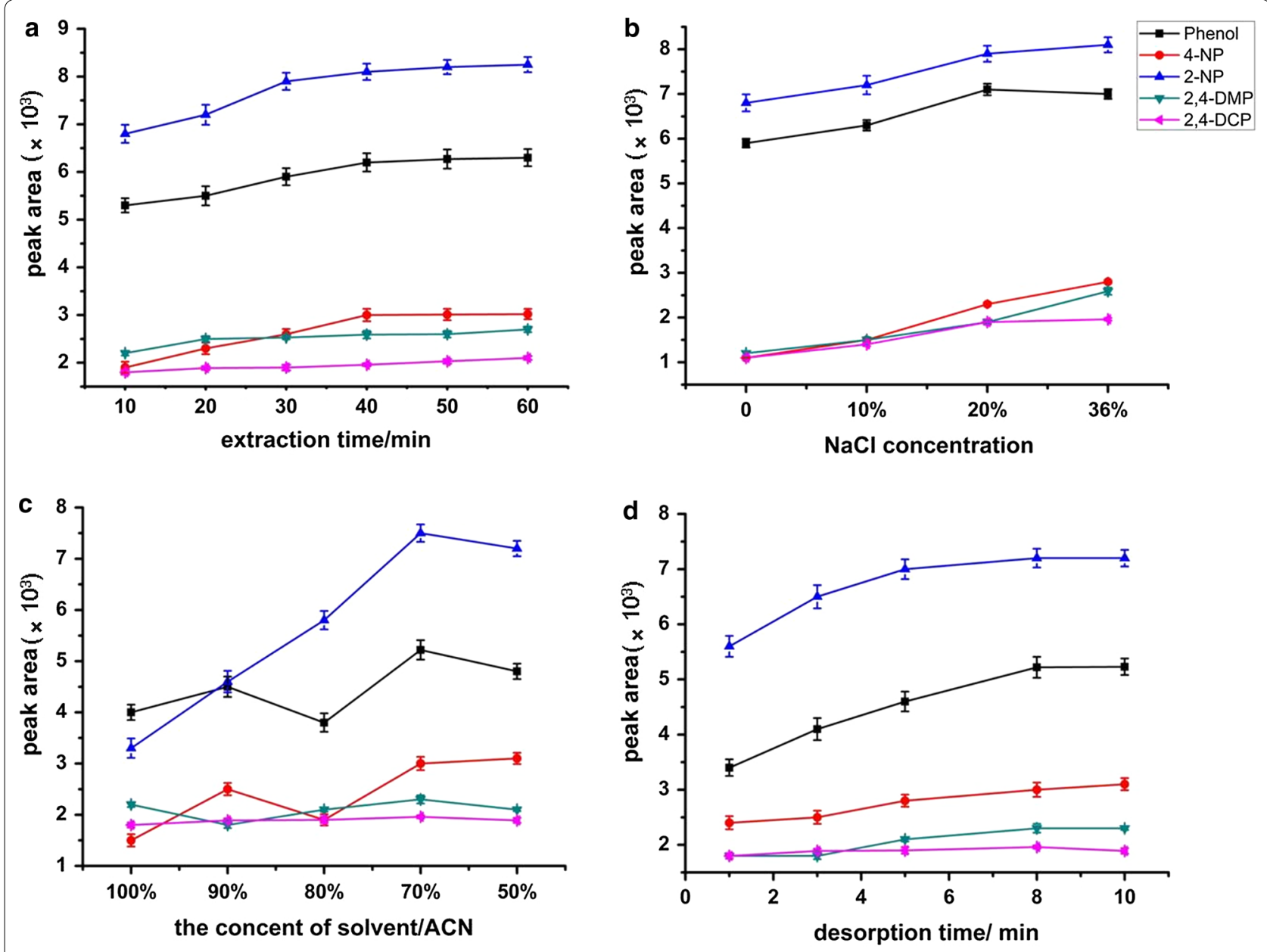

Fig. 4 Effects of the experimental conditions on the extraction efficiency of the MWCNTs/NaDC SPME fiber for $100 \mathrm{ng} / \mathrm{mL}$ each analyte. a Extraction time, $\mathbf{b}$ ionic strength, $\mathbf{c}$ the composition of the elution solvent, and $\mathbf{d}$ desorption time. Errors bars show the standard deviation of the mean $(n=3)$

\section{Desorption time}

The effect of desorption time was investigated with ultrasonication for between 1 and $10 \mathrm{~min}$ (Fig. 4d). All the phenols were desorbed almost completely within 8 min. Increasing the time above 8 min did not considerably increase the desorption efficiency. Therefore, 8 min was chosen as the optimum desorption time.

The optimum conditions for SPME of phenols were an extraction time of $50 \mathrm{~min}, \mathrm{NaCl}$ content of $36 \%$ $(\mathrm{w} / \mathrm{v}), \mathrm{ACN}$ volume fraction of $70 \%$, and desorption time of $8 \mathrm{~min}$.

\section{Comparison of the MWCNTs/NaDC fiber with a commercial PDMS/DVB fiber}

The extraction performance of the MWCNTs/NaDC fiber was compared with a commercial PDMS/DVB fiber ( $1 \mathrm{~cm}$ length, $65 \mu \mathrm{m}$ thick), which was suitable for the SPME of polar and half volatile compounds such as amines, phenols and parabens, etc. [44]. The SPME using the MWCNTs/NaDC and PDMS/DVB fiber was carried out with a phenol concentration of $100.0 \mathrm{ng} /$ $\mathrm{mL}$. The results are represented in Fig. 5. The relative response of the MWCNTs/NaDC fiber was higher than that of the commercial PDMS/DVB fiber for phenols. The high selectivity of the coating for phenols can be attributed to the hydrogen interactions with the analytes and the large surface area of the MWCNTs, which facilitated the phenols adsorption.

\section{Comparison of MWCNTs/NaDC fiber with MWCNTs fiber}

To evaluate the usefulness of the MWCNTs/NaDC Fiber, the adsorption efficiency of MWCNTs/NaDC with pristine MWCNTs were compared. The SPME analysis of the aqueous sample was executed by testing the sample spiked with phenols at $100.0 \mathrm{ng} / \mathrm{mL}$. As presented in Fig. 6, the peak area of the fiber with MWCNTs/NaDC 


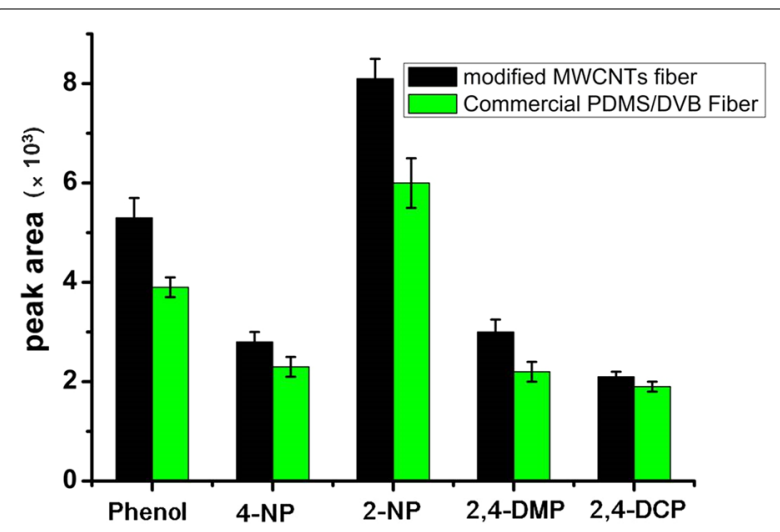

Fig. 5 Peak areas obtained with the MWCNTs/NaDC fiber and commercial PDMS/DVB fiber for phenols. The extraction of MWCNTs/ $\mathrm{NaDC}$ fiber and commercial PDMS/DVB fibers for phenols were under the optimized conditions. Extraction time: 50 min; stirring speed: $1100 \mathrm{rpm} ; \mathrm{NaCl}$ concentration: 36\% (w/v); concentration of phenols: $100.0 \mathrm{ng} / \mathrm{mL}$ for Phenol, 4-NP, 2-NP, 2,4-DMP and 2,4-DCP. Error bars show the standard deviation of the mean $(n=3)$

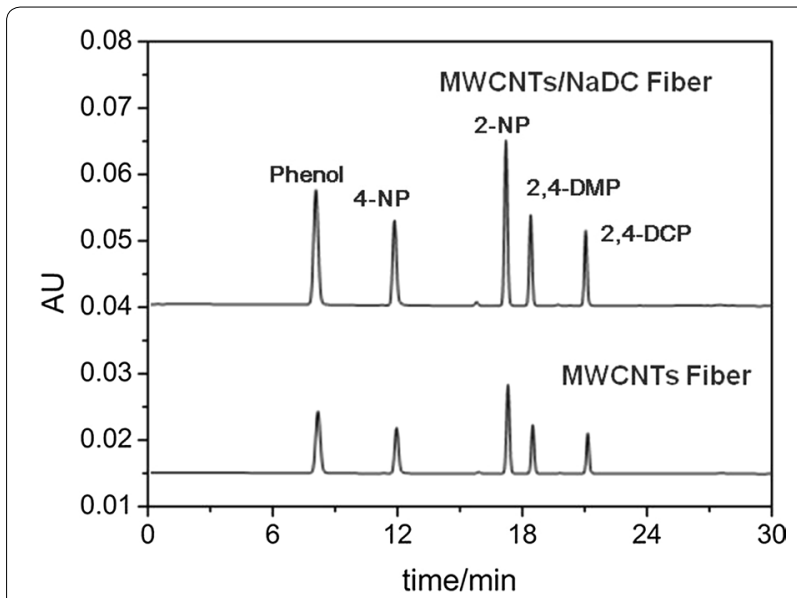

Fig. 6 Chromatograms of the phenols standard solution containing $100 \mathrm{ng} / \mathrm{mL}$ obtained with the MWCNTs/NaDC and the pristine MWCNTs fibers

increased additionally as compared to the pristine MWCNTs fiber, so that the better performance of the MWC$\mathrm{NTs} / \mathrm{NaDC}$ fiber was achieved. This can be explained by the fact that the modification of MWCNTs with NaDC increased the conjugation interaction between the coating and the target phenols. And the performance of MWCNT-coated SPME fiber can be enhanced by the complete dispersion and proper assembly of the nanotubes [45].

\section{The stability of MWCNTs/NaDC coating}

The stability of MWCNTs/NaDC coating is very important for practical applications. To check the stability of the extractive phase, the residual amount of $\mathrm{NaDC}$ in aqueous solution after the analyte extraction was determined. Figure 7 showed the chromatograms of $\mathrm{NaDC}$ standard solution and sample after the analyte extraction, almost no $\mathrm{NaDC}$ was found in aqueous solution, which demonstrates that the stability of MWCNTs/NaDC coating was good, no leaching of $\mathrm{NaDC}$ from the fiber to the water sample appeared.

\section{The lifetime of the coating}

The lifetime of a coating is also very important for practical applications. The reproducibility of the MWCNTs/ $\mathrm{NaDC}$ fiber extraction was investigated. After repeating phenol extraction and elution 20 times, the peak areas in the SPME chromatograms did not change remarkably (Fig. 8). These results demonstrated that there was no apparent loss in performance of MWCNTs/NaDC fiber after 20 times of extraction and desorption cycles, indicating that the coating is quite stable and reproducible.

\section{Method validation and application to real samples}

Under the optimized conditions, calibration curves were constructed for phenols analysis (Table 2). The linear ranges for all tested analytes were $1-100 \mathrm{ng} / \mathrm{mL}$ with good correlation coefficients $\left(R^{2}>0.9997\right)$. The limits of detection (LODs) were calculated at a signal-to-noise ratio of three, and the LOD ranges were $0.15-0.30 \mathrm{ng} /$ $\mathrm{mL}$. The LOQ were estimated from the $\mathrm{S} / \mathrm{Ns}$ of 10 , and LOQ values were in the range of $0.60-1.20 \mathrm{ng} / \mathrm{mL}$. The results showed that the RSD range between 4.9 and $10.2 \%$ from single fiber, and range between 5.7 and $11.9 \%$ from fiber to fiber with three replicates.

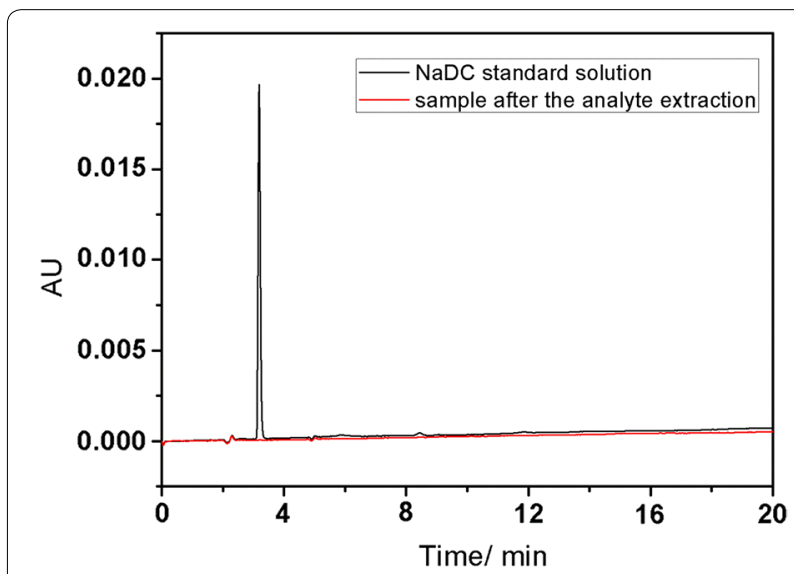

Fig. 7 Chromatograms of NaDC standard solution and sample after the analyte extraction.(Agela C18 column( $250 \mathrm{~mm} \times 4.6 \mathrm{~mm}$ i.d); The mobile phase was methyl alcohol: water (3:7) at a flow rate of $1.0 \mathrm{~mL} /$ min; The column temperature was $30^{\circ} \mathrm{C}$ and the wavelength was $210 \mathrm{~nm})$ 


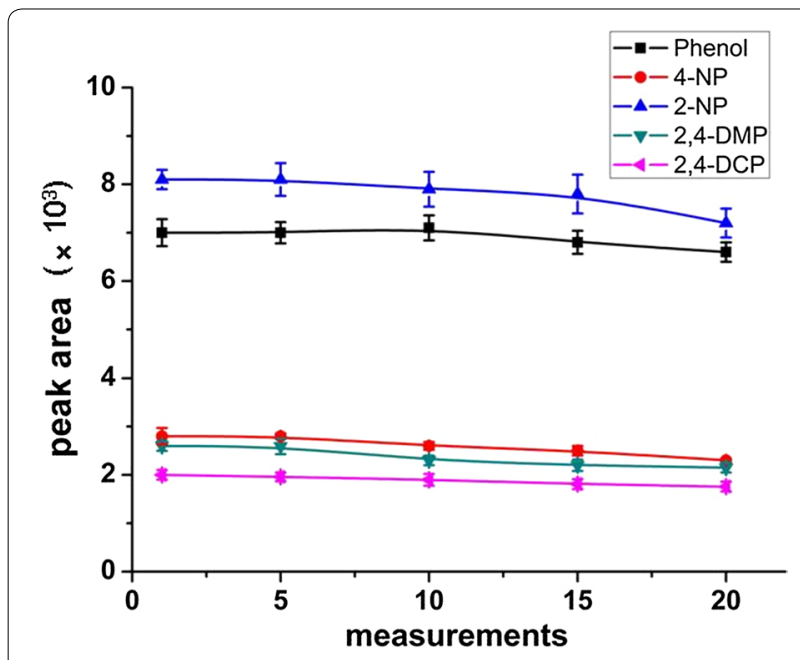

Fig. 8 Peak areas of the phenols as the function of the number of measurements performed with a single MWCNTs/NaDC fiber

The comparison of the proposed method with other methods was summarized in Table 3, which including SPME, Stir bar sorptive extraction (SBSE), Stir barsupported micro-solid-phase extraction (SB- $\mu$-SPE) and et al. The LODs of the developed method were lower than that obtained by MWCNTs-COOH fiber SPME [6] and Stir bar-supported micro-solid-phase extraction [47], and comparable with that achieved by MWCNTs-DDM/ PDMS SBME [38], PDMS-SBSE [16] and SB- $\mu$-SPE [46]. Although the LODs of this method were higher than that obtained by PDMS-SBSE [16], the RSD of this method were lower. In short, our method is comparable to existing methods and allows for trace analysis in real samples.

Then, the optimized method was applied to the determination of phenols in samples from the South China Sea and Wastewater. $10 \mathrm{~mL}$ of aqueous solution was extracted by this novel MWCNTs/NaDC fiber without any pretreatment, the results indicated that Phenol was detected in the wastewater $(3.64 \mathrm{ng} / \mathrm{mL})$, while the concentrations of 2-NP, 4-NP, 2,4-DMP and 2,4-DCP were below the LODs. No phenolic compounds were found in South China Seawater. Spiking experiments were then performed to evaluate the accuracy of the established method, and the samples were spiked with phenols at 10.0 and $100.0 \mathrm{ng} / \mathrm{mL}$. Figure 9 showed the chromatograms of phenols extracted by the MWCNTs/NaDC fiber from real water sample and spiked samples. As listed in Table 4, the recovery ranges were $85.6-93.1 \%$

Table 2 Analytical performance for HPLC determination of phenols using the MWCNTs/NaDC fiber

\begin{tabular}{llllllr}
\hline Compound & Linear equation & $\boldsymbol{R}^{\mathbf{2}}$ & LOD $(\mathbf{n g} / \mathbf{m L})$ & LOQ $(\mathbf{n g} / \mathbf{m L})$ & RSD $(\%, n=6)^{\mathbf{a}}$ & RSD $(\%, n=3)^{\mathbf{b}}$ \\
\hline Phenol & $y=54409 x+1438.9$ & 0.9997 & 0.16 & 0.64 & 4.9 & 7.6 \\
4-NP & $y=32887 x+368.02$ & 0.9997 & 0.28 & 1.12 & 5.1 & 11.9 \\
2-NP & $y=52340 x+711.27$ & 0.9998 & 0.15 & 0.60 & 6.3 & 8.8 \\
2,4-DMP & $y=26372 x+775.15$ & 0.9998 & 0.30 & 1.20 & 10.2 & 9.6 \\
2,4-DCP & $y=21672 x+224.74$ & 0.9998 & 0.29 & 1.16 & 7.1 & 5.7 \\
\hline
\end{tabular}

a Single fiber with six replicates

b Fiber to fiber with three replicates. The Linear ranges were $1-100 \mathrm{ng} / \mathrm{mL}$ for five phonels

Table 3 Comparison of the proposed method with other methods

\begin{tabular}{|c|c|c|c|c|c|}
\hline Method & Detection system & LOD (ng/mL) & RSD (\%) & Liner range $(\mathrm{ng} / \mathrm{mL})$ & References \\
\hline SPME $^{\mathrm{a}}$ & HPLC-UV & $0.25-3.67$ & $4.25-12.95$ & $10.8-1585$ & [6] \\
\hline SPME $^{b}$ & GC-MSMS & $0.26-2.63$ & $2.08-9.02$ & $1-1000$ & {$[21]$} \\
\hline MWCNTS-DDM/PDMS SBSEC & HPLC-UV & $0.14-1.76$ & $6.2-11.6$ & $1-1000$ & [38] \\
\hline PDMS-SBSE $^{d}$ & GC-MS & $0.1-0.4$ & $6-27$ & $1-15$ & [16] \\
\hline $\mathrm{SB}-\mu-\mathrm{SPE}^{\mathrm{e}}$ & GC-MS & 0.24 & 4.50 & $1-600$ & [46] \\
\hline SUPRAS-microextraction ${ }^{f}$ & HPLC-DAD & $1-4$ & $4.70-7.27$ & $10-150$ & [47] \\
\hline MWCNTS/NaDC SPME & HPLC-UV & $0.15-0.30$ & $5.7-11.9$ & $1-100$ & Present study \\
\hline
\end{tabular}

\footnotetext{
a MWCNTs-COOH fiber SPME

b Carboxylated solid carbon spheres SPME

c Amino modified multi-walled carbon nanotubes/polydimethylsiloxane coated stir bar sorptive extraction

d Polydimethyl siloxane (PDMS) stir bar sorptive extraction

e Stir bar-supported micro-solid-phase extraction

f Supramolecular solvent based microextraction
} 


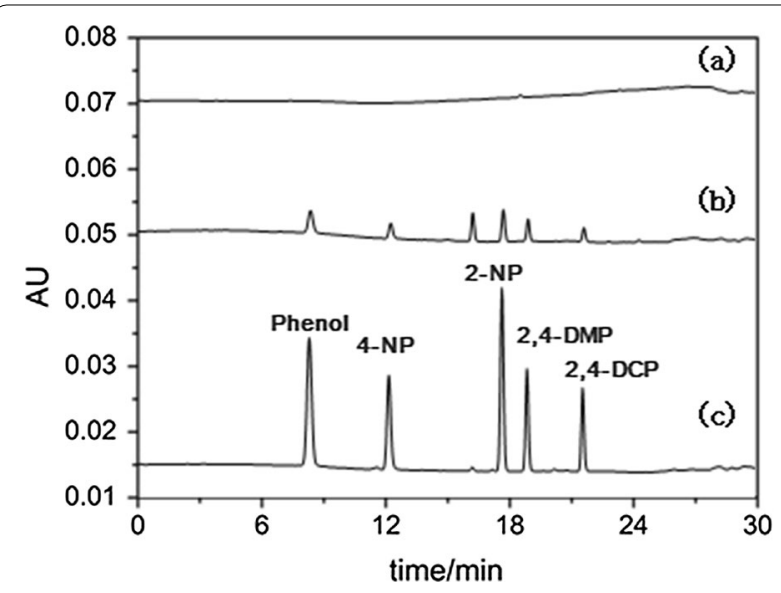

Fig. 9 Chromatograms of phenols extracted by the MWCNTs/NaDC fiber from (a) a blank Yangpu seawater sample and (b) the same seawater sample spiked with a phenols standard solution at $10.0 \mathrm{ng} /$ $\mathrm{mL}$. (c) The $100.0 \mathrm{ng} / \mathrm{mL}$ phenols standard solution used for spiking

Table 4 Analytical results for the determination of phenols in seawater samples from the South China Sea and Wastewater

\begin{tabular}{|c|c|c|c|c|c|}
\hline Samples & Phenol & 4-NP & 2-NP & 2,4-DMP & 2,4-DCP \\
\hline \multicolumn{6}{|l|}{ Yangpu } \\
\hline $\begin{array}{l}\text { Concentration (ng/ } \\
\mathrm{mL} \text { ) }\end{array}$ & $n d^{c}$ & nd & nd & nd & nd \\
\hline Recovery ${ }^{a}$ & 93.1 & 85.6 & 92.6 & 89.3 & 91.2 \\
\hline $\operatorname{RSD}(\%, n=3)$ & 4.2 & 5.0 & 6.8 & 5.1 & 4.9 \\
\hline \multicolumn{6}{|l|}{ Baishamen } \\
\hline $\begin{array}{l}\text { Concentration (ng/ } \\
\mathrm{mL} \text { ) }\end{array}$ & nd & nd & nd & nd & nd \\
\hline Recovery ${ }^{b}$ & 95.1 & 89.6 & 94.2 & 88.6 & 95.4 \\
\hline $\operatorname{RSD}(\%, n=3)$ & 6.4 & 5.9 & 6.8 & 7.1 & 8.2 \\
\hline \multicolumn{6}{|l|}{ Holiday beach } \\
\hline $\begin{array}{l}\text { Concentration (ng/ } \\
\text { mL) }\end{array}$ & nd & nd & nd & nd & nd \\
\hline Recovery ${ }^{\mathrm{b}}$ & 81.6 & 85.4 & 87.3 & 81.9 & 80.3 \\
\hline $\operatorname{RSD}(\%, n=3)$ & 5.9 & 6.8 & 7.1 & 6.9 & 8.3 \\
\hline \multicolumn{6}{|l|}{ Wastewater } \\
\hline $\begin{array}{l}\text { Concentration (ng/ } \\
\mathrm{mL} \text { ) }\end{array}$ & $3.64 \pm 0.17$ & nd & nd & nd & nd \\
\hline Recovery ${ }^{b}$ & 85.3 & 84.1 & 85.6 & 90.5 & 82.6 \\
\hline $\operatorname{RSD}(\%, n=3)$ & 5.9 & 6.8 & 7.1 & 6.9 & 8.3 \\
\hline
\end{tabular}

a Spiked with phenols at $10.0 \mathrm{ng} / \mathrm{mL}$

b Spiked with phenols at $100.0 \mathrm{ng} / \mathrm{mL}$

c $n d=$ not detected. Three parallel experiments were conducted for each sample (relative standard deviation $<7 \%$ ) for seawater samples from the Yangpu area, 88.6-95.4\% for samples from the Baishamen area, 80.3-87.3\% for samples from the Holiday Beach area, and $82.6-90.5 \%$ for wastewater samples. The precision and accuracy of the present method were acceptable.

\section{Conclusions}

In this work, a new MWCNTs/NaDC SPME fiber coupled with HPLC for trace analysis of phenols has been developed. The MWCNTs/NaDC fiber exhibited high extraction efficiencies, a wide linear range, low LODs and satisfactory reproducibility for phenols. The high adsorption capacity can be attributed to hydrogen bonding between the hydroxyl and carboxyl groups of the MWCNTs/NaDC surface and phenols. The mutual effect between the MWCNTs and sodium deoxycholate changed the electrical and steric resistance of the MWCNTs, and improved their solubility and dispersibility. The proposed fiber has better detection sensitivity than commercial PDMS/DVB fibers. The LOD range of the proposed method for phenols analysis was $0.15-0.30 \mathrm{ng} /$ $\mathrm{mL}$. These results broaden the potential for application of MWCNTs in the analysis of trace compounds in aqueous samples.

\section{Supplementary information}

Supplementary information accompanies this paper at https://doi. org/10.1186/s13065-020-00665-7.

Additional file 1. Additional tables.

\section{Acknowledgements}

We thank Gaobo Yu, Mingnan Zhang and analytical and testing centre of Hainan University for the technical support.

\section{Authors' contributions}

XZ conceived and designed the experiments. XZ and WF conducted the experiments and analysed the data. YX and ZZ analyzed the data. XZ wrote the paper. All authors read and approved the final manuscript.

\section{Funding}

This work was supported by the Hainan provincial natural science foundation (Grant No. 218QN186)

\section{Availability of data and materials}

All data generated or analysed during this study are included in this published article and its additional files.

\section{Competing interests}

The authors declare that they have no competing interest. 


\section{Author details}

${ }^{1}$ Analytical and Testing Center, Hainan University, Haikou 570228, China. ${ }^{2}$ College of Materials and Chemical Engineering, Hainan University, Haikou 570228, China.

Received: 14 July 2019 Accepted: 30 January 2020 Published online: 19 February 2020

\section{References}

1. The list of priority substances in the field of water policy and amending directive, Council directive 2455/2001/ECC, Official Journal L331, November 20 (2001) 1

2. Busca G, Berardinelli S, Resini C, Arrighi L (2008) Technologies for the removal of phenol from fluid streams: a short review of recent developments. J Hazard Mater 160:265-288

3. UNICEF and World Health Organization, Progress on Drinking-Water and Sanitation: 2012 Update, 2012

4. Li H, Wan L, Chu G, Tan W, Liu B, Qin Y, Feng Y, Sun D, Fang Y (2017) (Liquid + liquid) extraction of phenols from aqueous solutions with cineole. J Chem Thermodynam 107:95-103

5. Sarafraz-Yazdi A, Amiri A (2010) Liquid-phase microextraction. Trends Anal Chem 29:1-14

6. Liu X, Ji Y, Zhang Y, Zhang H, Liu M (2007) Oxidized multiwalled carbon nanotubes as a novel solid-phase microextraction fiber for determination of phenols in aqueous samples. J Chromatogr A 1165:10-17

7. Li QL, Wang XF, Yuan DX (2009) Preparation of solid-phase microextraction fiber coated with single-walled carbon nanotubes by electrophoretic deposition and its application in extracting phenols from aqueous samples. J Chromatogr A 1216:1305-1311

8. Zhou FR, Li X, Zeng ZR (2005) Determination of phenolic compounds in wastewater samples using a novel fiber by solid-phase microextraction coupled to gas chromatography. Anal Chim Acta 538:63-70

9. Abolghasemi MM, Yousefia V, Amirshaghaghi A (2015) Preparation and evaluation of a layered double hydroxide film on a nanoporous anodic aluminum oxide/aluminum wire as a highly thermal-resistant solid-phase microextraction fiber. New J Chem 39:3109-3115

10. Korba K, Pelit L, Pelit FO, Özdokur KV, Ertas H, Eroglu AE, Ertas F (2013) Preparation and characterization of sodium dodecyl sulfate doped polypyrrole solid phase micro extraction fiber and its application to endocrine disruptor pesticide analysis. J Chromatogr B 929:90

11. Xiao Z, Zhou X, Niu Y, Yu D, Zhu J, Zhu G (2015) Optimization and application of headspace-solid-phase micro-extraction coupled with gas chromatography-mass spectrometry for the determination of volatile compounds in cherry wines. J Chromatogr B 978-979:122-130

12. Spietelun A, Kloskowski A, Chrzanowsk W, Namieśnik J (2013) Understanding solid-phase microextraction: key factors influencing the extraction process and trends in improving the technique. Chem Rev 113:1667-1685

13. Gonzalez-Toledo E, Prat MD, Alpendurada MF (2001) Solid-phase microextraction coupled to liquid chromatography for the analysis of phenolic compounds in water. J Chromatogr A 923:45-52

14. Peñalver A, Pocurull E, Borrull F, Marcé RM (2002) Solid-phase microextraction coupled to high-performance liquid chromatography to determine phenolic compounds in water samples. J Chromatogr A 953:79-87

15. Quintana JB, Rodil R, Muniategui-Lorenzo S, Lopez-Mahia P, PradaRodriguez D (2007) Multiresidue analysis of acidic and polar organic contaminants in water samples by stir-bar sorptive extraction-liquid desorption-gas chromatography-mass spectrometry. J Chromatogr A 1174:27-39

16. Montero L, Conradi S, Weiss H, Popp P (2005) Determination of phenols in lake and ground water samples by stir bar sorptive extraction-thermal desorption-gas chromatography-mass spectrometry. J Chromatogr A 1071:163-169

17. Yu Y, Liu H, Dai X, Cai H, Li C, Yu H (2010) Trace analysis of phenols and chlorophenols in water by in situ derivatization headspace solid-phase microextraction coupled with gas chromatography/mass spectrometry. Chin J Anal Chem 38(9):1243-1248

18. Haberhauer-Troyer C, Crnoja M, Rosenberg E, Grasserbauer M, Fresenius $J$ (2000) Surface characterization of commercial fibers for solid-phase microextraction and related problems in their application. Anal Chem 366:329-331

19. Hu X, Hu Y, Li G (2007) Development of novel molecularly imprinted solid-phase microextraction fiber and its application for the determination of triazines in complicated samples coupled with high-performance liquid chromatography. J Chromatogr A 1147:1-9

20. Abolghasemi MM, Karimi B, Yousefi V, Behzadnia H, Barzegar H, Piryaei M (2015) Ionic liquid-derived nano-fibrillated mesoporous carbon based on solid-phase microextraction fiber for the analysis of volatile organic compounds from aqueous solutions. New J Chem 39:6085-6091

21. Amanzadeh H, Yamini Y, Masoomi MY, Morsali A (2017) Nanostructured metal-organic frameworks, TMU-4, TMU-5, and TMU-6, as novel adsorbents for solid phase microextraction of polycyclic aromatic hydrocarbons. New J Chem 41:12035-12043

22. Kueseng P, Pawliszyn J (2013) Carboxylated multiwalled carbon nanotubes/polydimethylsiloxane, a new coating for 96-blade solid-phase microextraction for determination of phenolic compounds in water. J Chromatogr A 1317:199-202

23. Gong SX, Wang X, Chen Y, Cheng CG, Wang ML, Zhao RS (2015) Carboxylated solid carbon spheres as a novel solid-phase microextraction coating for sensitive determination of phenols in environmental water samples. J Chromatogr A 1401:17-23

24. Wang FX, Zheng J, Qiu JL, Liu SQ, Chen GS, Tong YX, Zhu F, Ouyang GF (2017) In situ hydrothermally grown TiO2@C core-shell nanowire coating for highly sensitive solid phase microextraction of polycyclic aromatic hydrocarbons. Appl Mater Interfaces 9:1840-1846

25. Wang JX, Jiang DQ, Gu ZY, Yan XP (2006) Multiwalled carbon nanotubes coated fibers for solid-phase microextraction of polybrominated diphenyl ethers in water and milk samples before gas chromatography with electron-capture detection. J Chromatogr A 1137:8-14

26. Kong J, Franklin NR, Zhou C, Chapline MG, Peng S, Cho K, Dai H (2000) Nanotube molecular wires as chemical sensors. Science 287:622-625

27. Liu C, Fan YY, Liu M, Cong HT, Cheng HM, Dresselhaus MS (1999) Hydrogen storage in single-walled carbon nanotubes at room temperature. Science 286:1127-1129

28. Iijima S (1991) Helical microtubules of graphitic carbon. Nature 354:56-58

29. Iijima S, Ichihashi T (1993) Single-shell carbon nanotubes of 1-nm diameter. Nature 363:603-605

30. Basheer C, Alnedhary AA, MadhavaRao BS, Valliyaveettil S, Lee HK (2006) Development and application of porous membrane-protected carbon nanotube micro-solid-phase extraction combined with gas chromatography/mass spectrometry. Anal Chem 78:2853-2858

31. Abolghasemi MM, Yousefi V, Piryaei M (2015) Synthesis of carbon nanotube/layered double hydroxide nanocomposite as a novel fiber coating for the headspace solid-phase microextraction of phenols from water samples. J Sep Sci 38:1344-1350

32. Rastkari N, Ahmadkhaniha R, Yunesian M (2009) Single-walled carbon nanotubes as an effective adsorbent in solid-phase microextraction of low level methyl tert-butyl ether, ethyl tert-butyl ether and methyl tertamyl ether from human urine. J Chromatogr B 877:1568-1574

33. Ma JP, Xiao RH, Li JH, Yu JB, Zhang YQ, Chen LX (2010) Determination of 16 polycyclic aromatic hydrocarbons in environmental water samples by solid-phase extraction using multi-walled carbon nanotubes as adsorbent coupled with gas chromatography-mass spectrometry. J Chromatogr A 1217:5462

34. Cai Y, Jiang G, Liu J, Zhou Q (2003) Multi-walled carbon nanotubes packed cartridge for the solid-phase extraction of several phthalate esters from water samples and their determination by high performance liquid chromatography. Anal Chim Acta 494:149-156

35. Li QL, Yuan DX, Lin QM (2004) Evaluation of multi-walled carbon nanotubes as an adsorbent for trapping volatile organic compounds from environmental samples. J Chromatogr A 1026:283-288

36. Wang X, Li XJ, Li Z, Zhang YD, Bai Y, Liu HW (2014) Online coupling of in-tube solid-phase microextraction with direct analysis in real time mass spectrometry for rapid determination of triazine herbicides in water using carbon-nanotubes-incorporated polymer monolith. Anal Chem 86:4739-4747

37. Cai Y, Jiang G, Liu J, Zhou Q (2003) Multiwalled carbon nanotubes as a solid-phase extraction adsorbent for the determination of bisphenol $A$, 4-n-nonylphenol, and 4-tert-octylphenol. Anal Chem 75:2517-2521 
38. Cong Hu, Chen Beibei, He Man, Bin Hu (2013) Amino modified multiwalled carbon nanotubes/polydimethylsiloxane coated stir bar sorptive extraction coupled to high performance liquid chromatography-ultraviolet detection for the determination of phenols in environmental samples. J Chromatogr A 1300:165-172

39. Ai Y, Wu M, Li L, Zhao F, Zeng B (2016) Highly selective and effective solid phase microextraction of benzoic acid esters using ionic liquid functionalized multiwalled carbon nanotubes-doped polyaniline coating. J Chromatogr A 1437:1-7

40. Geng Y, Liu MY, Li J, Shi XM, Kim JK (1876) Effects of surfactant treatment on mechanical and electrical properties of CNT/epoxy nanocomposites. Compos Part A: Appl Sci Manufac 2008:39

41. Pandey P, Mohanty S, Nayak SK (2014) Tailoring dispersion and interaction of MWNT in polymer nanocomposites, using Triton X-100 as nonionic surfactant. J Mater Eng Perform 23:4385-4393

42. Grossiord N, Loos J, Regev O, Koning CE (2006) Toolbox for dispersing nanotubes into polymers to get conductive nanocomposites. Chem Mater 18:1089

43. Yang K, Wu W, Jing Q, Zhu L (2008) Aqueous adsorption of aniline, phenol, and their substitutes by multi-walled carbon nanotubes. Environ Sci Technol 42:7931-7936
44. Pacheco-Fernandez I, Najafi A, Pino V, Anderson JL, Ayala JH, Afonso AM (2016) Utilization of highly robust and selective crosslinked polymeric ionic liquid-based sorbent coatings in direct-immersion solid-phase microextraction and high-performance liquid chromatography for determining polar organic pollutants in waters. Talanta 158:125-133

45. Vaisman L, Marom G, Wagner HD (2006) Dispersions of surface-modified carbon nanotubes in-soluble and water-insoluble polymers. Adv Funct Mater 16:357

46. Tanimu A, Jillani SMS, Alluhaidan AA, Ganiyu SA, Alhooshani K (2019) 4-phenyl-1,2,3-triazole functionalized mesoporous silica SBA-15 as sorbent in an efficient stir bar-supported micro-solid-phase extraction strategy for highly to moderately polar phenols. Talanta 194:377-384

47. Seebunrueng K, Dejchaiwatana C, Santaladchaiyakit Y, Srijaranai S (2017) Development of supramolecular solvent based microextraction prior to high performance liquid chromatography for simultaneous determination of phenols in environmental water. RSC Adv. 7:50143-50149

\section{Publisher's Note}

Springer Nature remains neutral with regard to jurisdictional claims in published maps and institutional affiliations.
Ready to submit your research? Choose BMC and benefit from:

- fast, convenient online submission

- thorough peer review by experienced researchers in your field

- rapid publication on acceptance

- support for research data, including large and complex data types

- gold Open Access which fosters wider collaboration and increased citations

- maximum visibility for your research: over $100 \mathrm{M}$ website views per year

At BMC, research is always in progress.

Learn more biomedcentral.com/submissions 